\title{
Notas sobre Acaulospora bireticulata Rothwell \& Trappe e primeiro registro de Acaulospora koskei Blask. para o Brasil
}

\author{
Bruno Tomio Goto ${ }^{1,5}$, Milene Moreira ${ }^{2}$, Siu Mui Tsai ${ }^{3}$, \\ Elke Jurandir Bran Nogueira Cardoso ${ }^{4}$ e Leonor Costa Maia ${ }^{1}$
}

Recebido em 5/10/2006. Aceito em 15/08/2007

\begin{abstract}
RESUMO - (Notas sobre Acaulospora bireticulata Rothwell \& Trappe e primeiro registro de Acaulospora koskei Blask. para o Brasil). O conhecimento sobre a distribuição de espécies de fungos micorrízicos arbusculares (FMAs) ainda é restrito e as variações morfológicas observadas em isolados de uma mesma espécie podem proporcionar subsídios adicionais para o entendimento dos caracteres usados na taxonomia do grupo. Os objetivos deste trabalho foram implementar dados sobre a ocorrência de Acaulospora koskei Blaszk. e Acaulospora bireticulata Rothwell \& Trappe encontradas em áreas de Floresta de Araucaria angustifolia em São Paulo, Brasil, bem como comparar a descrição dessas espécies com a de outras similares.
\end{abstract}

Palavras-chave: Glomeromycota, Araucaria angustifolia, fungo micorrízico, cultura armadilha

\begin{abstract}
Notes on Acaulospora bireticulata Rothwell \& Trappe and first record of Acaulospora koskei Blask. from Brazil). Our knowledge of species distribution in arbuscular mycorrhizal fungi (AMF) is still limited and morphological variations found in isolates of one species may provide additional insight for understanding the characters used in the taxonomy of this group. The aims of this work were to expand biogeographical data regarding Acaulospora koskei Blaszk. and Acaulospora bireticulata Rothwell \& Trappe, both found in an Araucaria Forest in São Paulo state, Brazil, as well as to compare the descriptions of these species with those of other similar AMF.
\end{abstract}

Key words: Glomeromycota, Araucaria angustifolia, arbuscular fungi, trap culture

\section{Introdução}

A taxonomia dos fungos micorrízicos arbusculares (FMAs) tem se baseado nas características morfológicas dos esporos, principalmente na estrutura da parede (Gerdemann \& Trappe 1974). Porém, algumas espécies apresentam descrições incompletas, com ausência de detalhes importantes, impedindo assim uma acurada identificação. As descrições passaram a ser apresentadas de forma mais sistemática quando Walker (1983) propôs uma terminologia específica para as estruturas que formavam a parede do esporo. Por outro lado, algumas espécies de FMAs foram redescritas, sendo utilizado como base o material tipo, como em Scutellospora aurigloba (Hall) Walker \& Sanders, Glomus macrocarpum Tul. \& Tul. e G. microcarpum Tul. \& Tul. (Walker \& Hall 1991; Berch \& Fortin 1983).
As espécies de FMAs apresentam variação em alguns aspectos taxonômicos tais como: cor, forma, dimensão do esporo e detalhes da parede dos esporos como fenótipo e espessura das camadas, que dependem da origem ou ontogenia de cada espécie (Morton 1985; Bentivenga et al. 1997; Walker \& Vestberg 1998). Além disso, descrições de novas ocorrências têm revelado a presença de camadas não observadas nas descrições originais, como em Acaulospora koskei Blasz. (http://invam.caf.wvu.edu) e Acaulospora bireticulata Rothwell \& Trappe (Blaszkowski 1997). Tais descrições complementam dados referentes às variações encontradas em isolados de uma mesma espécie, facilitando a posterior identificação.

Os objetivos deste estudo foram descrever e comparar esporos de A. koskei e A. bireticulata isolados em Florestas de Araucaria angustifolia (Bert.) O. Ktze., fornecendo novos dados referentes

\footnotetext{
1 Universidade Federal de Pernambuco, Departamento de Micologia, Av. Prof. Nelson Chaves s.n., Cidade Universitária, 50670-420 Recife, PE, Brasil

2 Instituto Agronômico, Centro de Solos e Recursos Ambientais, Av. Barão de Itapura 1481, 13001-970 Campinas, SP, Brasil

3 Universidade de São Paulo, Microbiologia e Biologia Molecular, CENA/USP, Av. Centenário 303, 13416-000 Piracicaba, SP, Brasil

4 Escola Superior de Agricultura “Luiz de Queiroz", Departamento de Solos e Nutrição de Plantas, Av. Pádua Dias 9, 13418-900 Piracicaba, SP, Brasil

5 Autor para correspondência: brunogoto@hotmail.com
} 
a variações nos caracteres morfológicos dessas espécies.

\section{Material e métodos}

Esporos de FMAs foram extraídos, por peneiramento úmido (Gerdemann \& Nicolson 1963) e centrifugação em água e sacarose (Jenkins 1964), do solo rizosférico de Araucaria angustifolia nativa do Parque Estadual de Campos do Jordão (2244'S e 4530’W), Município de Campos do Jordão, Estado de São Paulo, Brasil. Coletaram-se amostras de solo na profundidade de $0-20 \mathrm{~cm}$ em maio/2002, em três subáreas, uma de floresta nativa e duas de replantio. Em cada subárea, com dimensão de aproximadamente $1.000 \mathrm{~m}^{2}$, foram escolhidas ao acaso cinco árvores; em cada árvore foram amostrados três pontos, eqüidistantes entre si, onde foram coletadas $300 \mathrm{~g}$ de solo rizosférico. O conjunto (três pontos/árvore) constituiu uma amostra composta. Os esporos, separados em grupos de acordo com as características morfológicas, foram montados em lâminas semipermanentes, com resina de ácido lático, álcool polivinílico e glicerol (PVLG) e com reagente de Melzer + PVLG (1:1) (Morton et al. 1993) e observados em microscópio de luz, sendo identificados com auxílio do manual de Schenck \& Pérez (1990), descrições fornecidas pelo INVAM (http://invam.caf.wvu.edu) e literatura pertinente.

Foram preparadas culturas armadilha utilizando-se como plantas hospedeiras, milho (Zea mays L.) e amendoim (Arachis hypogaea L.) num mesmo vaso, e plantas de araucária, noutro vaso. O substrato usado para plantio foi proveniente de uma amostra de Neossolo Quartzarênico (série Paredão Vermelho) + areia de rio lavada, na proporção de $2: 3(\mathrm{v}: \mathrm{v})$, com as seguintes características: $\mathrm{pH}$ em $\mathrm{CaCl}_{2} 4,0 ; 13 \mathrm{~g} \mathrm{dm}^{-3}$ de M.O.; $3 \mathrm{mg} \mathrm{dm}^{-3}$ de P (resina); 1,4 ; 4; 3; 6 e 31 $\mathrm{mmol}_{\mathrm{c}} \mathrm{dm}^{-3}$ de $\mathrm{K}^{+}, \mathrm{Ca}^{+2}, \mathrm{Mg}^{+2}, \mathrm{Al}^{+3} \mathrm{e}^{+}+\mathrm{Al}^{+3}$, respectivamente. Este substrato foi seco, peneirado e autoclavado à $121{ }^{\circ} \mathrm{C}$, por duas horas, sendo posteriormente colocado em vasos de $6 \mathrm{~L}$ de capacidade para as plantas de milho e amendoim e em vasos de $20 \mathrm{~L}$ para as plantas de araucária, onde foram incorporados $150 \mathrm{~g}$ de inóculo (contendo propágulos de FMA) preparado a partir de cada uma das amostras compostas, obtidas em cada subárea. Foram utilizados dez vasos por subárea, metade com consórcio de milho e amendoim e metade com araucária. As sementes de milho e amendoim foram desinfestadas superficialmente com solução de hipoclorito de sódio a $2 \%$, por cinco minutos e as de araucária foram escarificadas (Moreira-Souza \& Cardoso 2003). Dez dias após a semeadura do milho e do amendoim, fez-se o desbaste, mantendo-se a planta mais vigorosa de cada espécie. As culturas armadilha foram mantidas em casa de vegetação por 160 dias (milho e amendoim) e 12 meses (araucária), sendo depois extraídos os esporos de FMAs para estudo morfológico e identificação das espécies. A terminologia dos esporos foi a sugerida por Goto \& Maia (2006).

\section{Resultados e discussão}

Acaulospora koskei Blaszk., Mycol. Res. 99: 237. 1995.

Fig. 1-2

Glomerosporos globosos a subglobosos (142,5-)167,3 (-187,5) $\mu \mathrm{m}$, de coloração amarelo claro a amarelo escuro, levemente alaranjada em PVLG. Parede do esporo composta de seis camadas, sendo a primeira evanescente, hialina $<1,0 \mu \mathrm{m}$ de espessura observada normalmente em esporos jovens; vestígios podem ser vistos em esporos maduros; segunda camada laminada, amarelo claro em PVLG e amarelo amarronzado em Melzer, até 2,5 $\mu \mathrm{m}$ de espessura; terceira camada hialina a levemente amarelada, laminada, com lâminas pouco evidentes, até $2,5 \mu \mathrm{m}$ de espessura, vermelha púrpura em Melzer; quarta camada membranosa, hialina, até 1,0 $\mu \mathrm{m}$ de espessura; quinta camada hialina, membranosa e fina, com granulações, fortemente aderida à sexta camada, que reage em Melzer adquirindo coloração vermelho púrpura, similar ao observado na terceira camada. Juntas, a quinta e a sexta camadas atingem até $2,5 \mu \mathrm{m}$ de espessura. Granulações da superfície da quinta camada raramente observadas nos esporos quebrados. Sáculo esporífero globoso, hialino, azulado em Melzer (amilóide). Cicatriz do sáculo esporífero facilmente visível na superfície dos esporos, às vezes formando um anel espesso (7,5 $\mu \mathrm{m}$ diâm.), decorrente de um espessamento da parede no local da inserção. Alguns esporos não apresentaram reação em Melzer, principalmente os jovens, que não tinham as camadas do componente germinativo desenvolvidas.

Em alguns esporos quebrados de $A$. koskei foram observados dobramentos da segunda camada (laminada) da parede similares aos referidos na superfície de A. rugosa Morton, porém diferenças na dimensão e coloração dos esporos, estrutura da parede e reação ao Melzer facilmente distinguem as duas 
espécies. Esporos de A. koskei podem ser confundidos com os de A. colossica Schultz, Bever \& Morton principalmente pela coloração que se superpõe (Schultz et al. 1999). Todavia, esporos de A. koskei são facilmente distinguidos pela reação ao Melzer em duas camadas da parede, enquanto isso não é observado em A. colossica. A coloração e a forma globosa a subglobosa também contribui para se confundir esporos de A. koskei com os de A. laevis Gerdemann \& Trappe. No entanto, os esporos de A. laevis $(119-300 \times 119-520 \mu \mathrm{m})$ são maiores que os de A. koskei e apresentam parede composta por três camadas (Gerdemann \& Trappe 1974), diferindo, portanto, da estrutura de parede de esporos de A. koskei, com seis camadas, em três grupos. Uma outra camada, de natureza membranosa, entre a camada laminada e a unitária, tem sido mencionada em esporos de A. koskei (http://invam.caf.wvu.edu), porém não foi observada nos esporos aqui descritos. O material avaliado nesse estudo apresenta características que se enquadram na descrição de Blaszkowski (1995), que menciona parede composta por apenas seis camadas.

Esporos de A. koskei não foram observados quando se fez extração direta do solo das amostras trazidas do campo, porém foram isolados a partir das culturas armadilhas em casa de vegetação, esporulando nas duas condições de cultivo (potes com milho/ amendoim e potes com araucária), confirmando a importância das culturas armadilha nos estudos de diversidade de FMAs.

Material examinado: BRASIL. São Paulo: Município de Campos do Jordão, V/2002, em potes de cultura armadilha com Zea mays, Arachis hypogaea e Araucaria angustifolia, M. Moreira (URM 45749).

Distribuição e habitat: conhecido para dunas na Polônia (Blaszkowski 1995), áreas alagadiças dos EUA (Miller \& Bever 1999) e na Inglaterra (Marryweather \& Fitter 1998) e em solos de Campos do Jordão, estado de São Paulo, sudeste do Brasil.

Acaulospora bireticulata Rothwell \& Trappe, Mycotaxon 8: 472. 1979.

Fig. 3-4

Glomerosporos globosos (130-)165,3(-187,5) $\mu \mathrm{m}$, coloração marrom escura ou verde escura, raramente laranja amarronzado em PVLG. Parede do esporo composta de sete camadas: a primeira evanescente e dificilmente observada, a segunda laminada, com 7,5-12,5 $\mu \mathrm{m}$ de espessura, ornamentada por retículos alveolados (circulares ou irregulares) ou poligonais (tetragonal, pentagonal ou hexagonal), correspondendo ao primeiro grupo (sensu Walker 1983). Espinhos de coloração amarela clara, inseridos nos retículos que medem de 1,5-2,5 $\mu \mathrm{m}$ larg. A terceira camada é hialina, membranosa, fina, fortemente aderida à quarta camada, também hialina e fina, as duas atingindo 2,0 $\mu \mathrm{m}$ de espessura e correspondendo ao segundo grupo de paredes (sensu Walker 1983). A quinta camada é hialina, 1,0 $\mu \mathrm{m}$, coriácea, fortemente aderida à sexta camada, 1,0 $\mu \mathrm{m}$, também hialina, juntas alcançando aproximadamente $2,0 \mu \mathrm{m}$ de espessura e com forte reação ao Melzer, adquirindo coloração vermelha púrpura. A sétima camada é hialina, fina $(<1,0 \mu \mathrm{m})$, não reage em Melzer e dificilmente é observada, mesmo em esporos maduros. Sáculo esporífero de coloração hialina a amarela clara, raramente preso ao esporo. Cicatriz normalmente circular, difícil de ser vista devido à ornamentação da camada externa da parede do esporo.

A presença de uma camada evanescente recobrindo os esporos de A. bireticulada tem sido referida (http://invam.caf.wvu.edu e http:// www.agro.ar.szczecin.pl/ jblaszkowski), porém apenas vestígios estavam presentes no material examinado.

Rothwell \& Trappe (1979) descreveram os esporos de A. bireticulata com coloração hialina a marrom claro e três camadas de parede. No entanto, em análise mais detalhada da parede do esporo, Blaszkowski (1997) descreveu a espécie admitindo a existência de sete camadas, distribuídas em três grupos. $\mathrm{Na}$ maioria dos esporos do isolado avaliado, apenas cinco camadas foram facilmente discerníveis, com a primeira e a última (evanescente e membranosa, respectivamente) sendo raramente observadas.

Glomerosporos de A. bireticulata são muito semelhantes aos de A. elegans Trappe \& Gerdemann, principalmente no que se refere à ornamentação da camada laminada, composta por espinhos dentro de um retículo alveolado ou poligonal. Segundo Gerdemann \& Trappe (1974), os esporos de A. elegans são globosos a subglobosos (140-285×145-330 $\mu \mathrm{m})$, elipsóides ou reniformes e têm parede com três camadas, enquanto os esporos de A. bireticulata são menores (130-)165,3(-187,5) $\mu \mathrm{m}$ e a parede é composta por maior número de camadas, em três grupos (Blaszkowski 1997).

Os esporos isolados da rizosfera de araucária diferem dos de $A$. elegans pelo tamanho (-130)165,3 (187,5-) $\mu \mathrm{m}$ e pela estrutura da parede. Morton (2005, http://invam.caf.wvu.edu) sugere que 


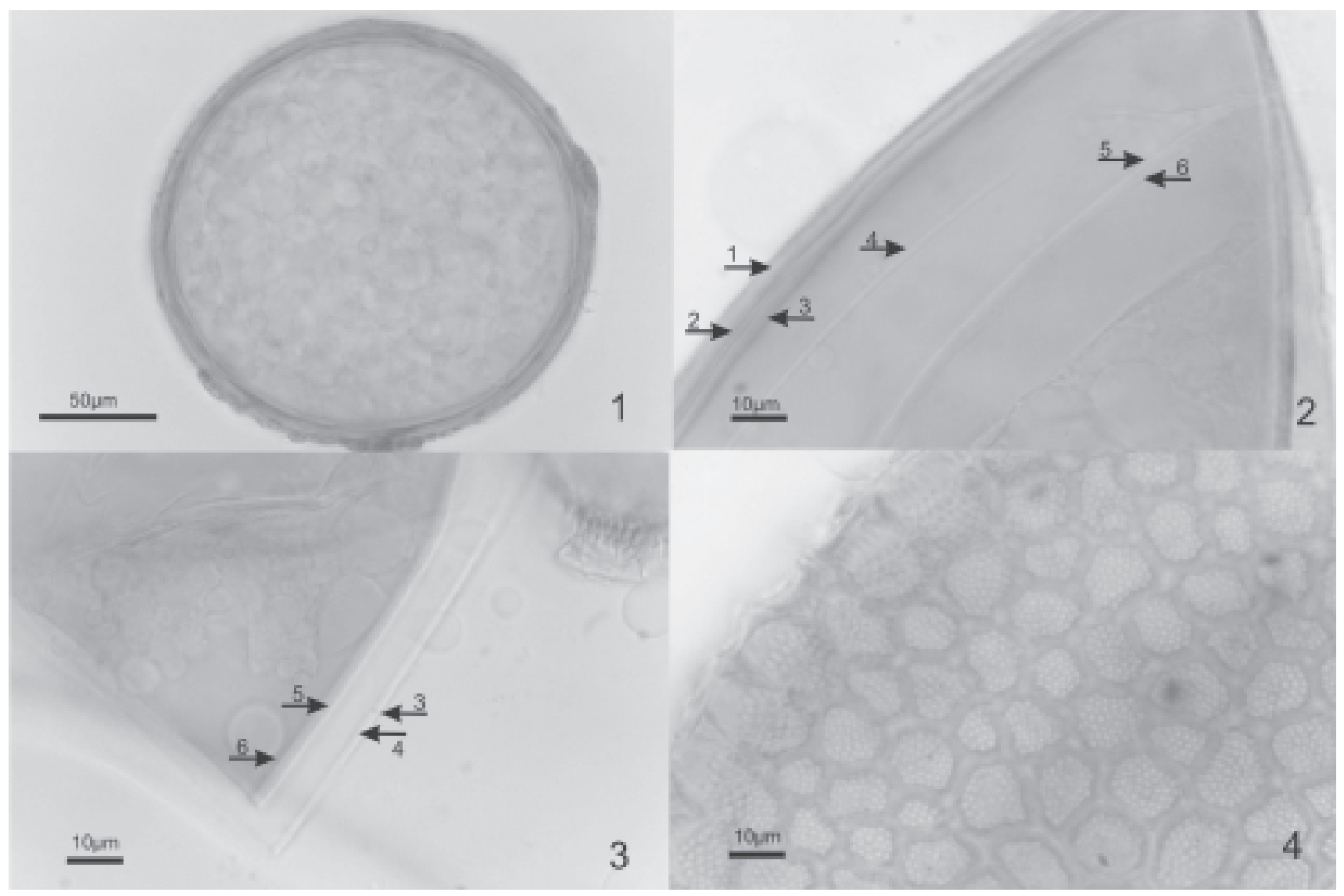

Figuras 1-4. Acaulospora koskei Blaszk. e Acaulospora bireticulata Rothwell \& Trappe. 1. Aspecto geral de um esporo de A. koskei. 2. Detalhe da estrutura da parede de esporos de A. koskei. 3. Detalhe da estrutura das camadas internas dos esporos de A. bireticulata. 4. Detalhe da ornamentação da parede de A. bireticulata.

A. bireticulata seja sinonímia de A. elegans, com base na sobreposição dos caracteres observados em isolados de ambas as espécies. Variações (plasticidade) entre os isolados podem ser evidências que corroboram esta hipótese. Entretanto, nenhum trabalho foi realizado com o material tipo para confirmar tal sinonimização. No material examinado, a camada laminada não foi observada em detalhes porque esse grupo de parede, onde estão as ornamentações, é resistente à pressão e dificilmente se separa. A sétima camada (do terceiro grupo), referida por Blaszkowski (1997) foi dificilmente visualizada, o que mostra a variabilidade na morfologia da parede de esporos de uma mesma espécie. No caso de A. bireticulata e A. elegans, muito semelhantes, mas ainda considerados táxons distintos, são necessários estudos ultraestruturais e moleculares para auxiliar na delimitação da identidade taxonômica desses dois fungos.

Esporos de A. bireticulata foram extraídos diretamente do solo coletado na área nativa e na reflorestada. O fungo também produziu esporos nas culturas armadilha, nas duas condições de cultivo.
Material examinado: BRASIL. São Paulo: Município de Campos do Jordão, V/2002, isolado de floresta nativa e de área reflorestada na rizosfera de Araucaria angustifolia e mantido em potes de cultura com Arachis hypogaea, M. Moreira (URM 45750).

Distribuição e habitat: conhecida em sistemas agrícolas, Floresta Atlântica e Floresta de Araucária e Pampas Argentinos (Maia \& Trufem 1990; Trufem \& Malatinszky 1995; Carrenho \& Trufem 2001; de Souza et al. 2002; Moreira-Souza et al. 2003; Focchi et al. 2004; Schalamuk et al. 2006), com registro também nos Estados Unidos (Rothwell \& Trappe 1979) e na Europa (Blaszkowski 1997).

\section{Agradecimentos}

À Fundação de Amparo à Pesquisa do Estado de São Paulo (FAPESP), pela bolsa de Pós-doutorado (proc. 01/13229-9) concedida a Milene Moreira e pelo suporte financeiro ao projeto Biota (proc. 01/05146-6); à CAPES e ao $\mathrm{CNPq}$, respectivamente, pela concessão de bolsa de Doutorado a B.T. Goto e de 
pesquisa a L.C. Maia, S.M. Tsai e E.J.B.N. Cardoso; à Diretoria do Parque de Campos do Jordão e à Secretaria do Meio Ambiente do Estado de São Paulo, por autorizar a coleta do material (proc. $40405 / 98$ COTEC 079/98).

\section{Referências bibliográficas}

Bentivenga, S.P.; Bever, J.D. \& Morton, J.B. 1997. Genetic variation of morphological characters within a single isolate of the endomycorrhizal fungus Glomus clarum (Glomaceae). American Journal of Botany 84: 1211-1216.

Berch, S.M. \& Fortin, J.A. 1983. Lectotypification of Glomus macrocarpum and proposal of three new combinations: Glomus australe, Glomus tenebrosum, and Glomus versiforme (Endogonaceae). Canadian Journal of Botany 61: 2608-2617.

Blaszkowski, J. 1995. Acaulospora koskei, a new species in Glomales from Poland. Mycological Research 99: 237-240.

Blaszkowski, J. 1997. Notes on Acaulospora bireticulata (Glomales, Zygomycetes) found in Poland. Mycotaxon 61: 193-204.

Carrenho, R. \& Trufem, S.F.B. 2001. Caracterização morfológica de esporos de fungos micorrízicos arbusculares isolados de solo cultivado com milho na Reserva Ecológica e Estação Experimental de MojiGuaçu, São Paulo, Brasil. Hoehnea 28: 191-208.

de Souza, P.V.; Schmitz, J.A.K.; Freitas, R.S. \& Carrenho, R. 2002. Identificação e quantificação de fungos micorrízicos arbusculares autóctones em municípios produtores de citrus no Rio Grande do Sul. Pesquisa Agropecuária Brasileira 37: 553-558.

Focchi, S.S.; dal Soglio, F.K.; Carrenho, R.; de Souza, P.V. \& Lovato, P.E. 2004. Fungos micorrízicos arbusculares em cultivos de citros sob manejo convencional e orgânico. Pesquisa Agropecuária Brasileira 39: 469-476.

Gerdemann, J.W. \& Nicolson, T.H. 1963. Spores of mycorrhizal Endogone species extracted from soil by wet sieving and decanting. Transactions of the British Mycological Society 46: 235-244.

Gerdemann, J.W. \& Trappe, J.M. 1974. The Endogonaceae in the Pacific Northwest. Mycologia Memoir 5: 1-76.

Goto, B.T. \& Maia, L.C. 2006. Glomerospores: a new denomination for the spores of Glomeromycota, a group molecularly distinct from the Zygomycota. Mycotaxon 96: $129-132$.

Jenkins, W.R. 1964. A rapid centrifugal-flotation technique for separating nematodes from soil. Plant Disease Report 48: $1-692$.

Maia, L.C. \& Trufem, S.F.B. 1990. Fungos micorrízicos vesículoarbusculares em solos cultivados no estado de Pernambuco, Brasil. Revista Brasileira de Botânica 13: 89-95.
Marryweather, J. \& Fitter, A. 1998. The arbuscular mycorrhizal fungi of Hyacinthoides non-scripta II. Seasonal and spacial patterns of fungal populations. New Phytologist 138: 131-142.

Miller, S.P. \& Bever, J.D. 1999. Distribution of arbuscular mycorrhizal fungi in stands of the wetland grass Panicum hemitomon along a wide hydrologic gradient. Oecologia 119: 586-592.

Moreira-Souza, M. \& Cardoso, E.J.B.N. 2003. A pratical method for germination of Araucaria angustifolia (Bert.) O. Ktze. Scientia Agricola 60: 389-391.

Moreira-Souza, M.; Trufem, S.F.B.; Gomes-da-Costa, S.M. \& Cardoso, E.J.B.N. 2003. Arbuscular mycorrhizal fungi associated with Araucaria angustifolia (Bert.) O. Ktze. Mycorrhiza 13: 211-215.

Morton, J.B. 1985. Variation in mycorrhizal and spore morphology of Glomus occultum and Glomus diaphanum as influenced by plant host and soil environment. Mycologia 77: 192-204.

Morton, J.B.; Bentivenga, S.P. \& Wheeler, W.W. 1993. Germ plasm in the International Collection of Arbuscular and Vesicular-Arbuscular Mycorrhizal Fungi (INVAM) and procedures for culture development, documentation and storage. Mycotaxon 48: 491-528.

Rothwell, F.M. \& Trappe, J.M. 1979. Acaulospora bireticulata sp. nov. Mycotaxon 8: 471-475.

Schalamuk, S.; Velazquez, S.; Chidichimo, H. \& Cabello, M. 2006. Fungal spore diversity of arbuscular mycorrhizal fungi associated with spring wheat: effects of tillage. Mycologia 98: 16-22.

Schenck, N.C. \& Peréz, Y. 1990. Manual for identification of VA mycorrhizal fungi. Gainesville, Synergistic Publications.

Schultz, P.A.; Bever, J.D. \& Morton, J.B. 1999. Acaulospora colossica sp. nov. from an old field in North Carolina and morphological comparisons with similar species, A. laevis and A. koskei. Mycologia 91: 676-683.

Trufem, S.F.B. \& Malatinszky, S.M.M. 1995. Fungos micorrízicos arbusculares em Melastomataceae e outras plantas nativas resistentes e sensíveis à poluição na Reserva Biológica do Alto da Serra de Paranapiacaba, SP, Brasil. Hoehnea 22: 77-89.

Walker, C. 1983. Taxonomic concepts in the Endogonaceae: spore wall characteristics in species descriptions. Mycotaxon 18: 443-455.

Walker, C. \& Hall, I. 1991. Lectotypification of Scutellospora auriglobosa (Glomales). Mycological Research 95: 398-400.

Walker, C. \& Vestberg, M. 1998. Synonymy amongst the arbuscular mycorrhizal fungi: Glomus claroideum, G. maculosum, G. multisubstensum and G. fistulosum. Annals of Botany 82: 601-624. 